\title{
Enhanced Aortic Pressure Wave Reflection in Patients with Aortic Coarctation after Aortic Arch Repair
}

\author{
Tomoaki Murakami \\ Department of Cardiology, Chiba Children's Hospital, Chiba, Japan
}

\section{Keywords}

Aortic coarctation - Pressure wave reflection - Cardiovascular disease - Hypertension - Pulse pressure amplification

\begin{abstract}
Background: In patients with aortic coarctation after successful aortic arch repair, it is well known that early-onset cardiovascular diseases can develop. Summary: We studied the pressure waveform in patients after aortic arch repair focusing on a pressure wave reflection. In patients after aortic arch repair, the repaired portion generates a new reflected pressure wave. As a result, the newly generated pressure wave causes aortic pressure augmentation, loss of pressure amplification, and left ventricular hypertrophy with fibrosis. Balloon dilatation of the aortic arch may also generate a new pressure wave reflection. Key Messages: In patients with aortic coarctation after aortic arch repair, the reconstructed site generates a new pressure wave reflection. This could be one of the causes of their future cardiovascular diseases.
\end{abstract}

(c) 2017 S. Karger AG, Basel

\section{Introduction}

Aortic coarctation is a congenital stenosis located in a juxta-ductal position of the aortic arch. Patients with aortic coarctation need surgical reconstruction or a catheter intervention (older children and adults) of their aortic arch. It is well known that subsequent cardiovascular complications have been frequently encountered despite an apparently successful surgical repair of the aortic coarctations [1-5]. Although many of the complications are related to anatomical problems (restenosis, aneurysm formation, and so forth), there are

Tomoaki Murakami, MD

Department of Cardiology, Chiba Children's Hospital

579-1, Heta-cho, Midori-ku

Chiba 266-0007 (Japan)

E-Mail murat @ seagreen.ocn.ne.jp 
some complications that are not directly related to the morphological problems of the reconstructed aorta. One of the complications is the early onset of cardiovascular diseases (hypertension, myocardial infarction, cardiac failure, and sudden death) [6-8]. It has been reported that these problems can even occur in patients with a completely satisfactory aortic arch repair. Cohen et al. [4] demonstrated that the most important predictor of long-term survival and hypertension in patients after aortic coarctation repair was the age at the time of the initial repair. These findings suggest that preoperative cardiovascular damage might have some unfavorable effects on the patient's postoperative condition. However, O'Sullivan et al. [8] reported that a high proportion of patients after an early and successful repair of aortic coarctation also showed hypertension. To explain this phenomenon, many hypotheses have been proposed, but it remains controversial.

Our studies have addressed this problem, and findings suggest that enhanced pressure wave reflection has a negative impact on patients after aortic arch repair. In this review, I will present features of pressure waveforms in patients after aortic arch repair and try to clarify the cause of cardiovascular diseases in patients after aortic arch repair based on pressure waveform analysis.

\section{Enhanced Aortic Pressure Wave Reflection in Patients after Aortic Arch Repair}

An aortic pressure waveform is composed of 2 pressure waveforms - a forward pressure waveform and a backward pressure waveform (a reflected pressure waveform) (Fig. 1). The forward pressure wave is generated by a left ventricular ejection, while the backward pressure wave is the sum of the pressure wave reflections. The pressure wave reflection causes an aortic pressure augmentation and is a physiologically important phenomenon, because the reflected pressure wave enhances coronary blood supply. However, it is well known that an excess of aortic pressure augmentation increases the risk of cardiovascular diseases [9-12]. The pressure wave reflections arise from discontinuities in the elastic properties along the arterial tree in which there is a change or mismatch in impedance [13]. In a normal aortic tree, it is reported that a reflecting point that represents the integrated pressure wave reflections exists around the region of an aortic bifurcation [9]. The reflected pressure wave returns to the heart during diastole (i.e., after a closure of the aortic valve) in young people, because of their slow pulse wave velocity. Therefore, the reflected pressure wave which returns to the ascending aorta during diastole enhances coronary perfusion by pushing the blood stored during systole in the aorta. With aging, the pulse wave velocity gradually increases, which means the early return of the reflected pressure wave (in systole), which impairs the arterial and ventricular functions. The opposite directional reflected pressure wave that returns to the heart in systole interferes with the left ventricular ejection and increases the workload of the left ventricle. In order to quantify the pressure wave reflection, an augmentation index is calculated (Fig. 1). The augmentation index is defined as the ratio of the augmentation pressure to the pulse pressure. The inflection point of the pressure waveform is defined as the timing with the peak flow velocity or obtained by the fourth derivative of the original pressure waveform. Besides the pulse wave velocity, many factors can influence the augmentation index. The distance from the heart to the inflection point is one of the important determinants of the augmentation index. It is reasonable that a short propagation distance means an early return of the reflected pressure wave. Practically, it has been reported that the augmentation index is high and inversely related to height in children [14]. When there is a strong inflection point closer to the heart than the normal inflection point (i.e., aortic bifurcation), the augmentation index measured at the ascending aorta should theoretically be high. 
Fig. 1. Aortic pressure waveform and augmentation index. An aortic pressure waveform consists of two pressure waveforms - a forward pressure waveform and a backward pressure waveform (a reflected pressure waveform). The contribution of the reflected pressure wave is quantified by an augmentation index.

Fig. 2. Pressure waveform in a 6-year-old subject with a normal aortic arch and a 6-year-old patient with aortic coarctation after a successful repair. In the normal aorta, the augmentation index of the ascending aortic pressure waveform is smaller than that of the descending aortic pressure waveform. On the other hand, the ascending aortic pressure waveform is almost identical to the descending aortic pressure waveform in patients after aortic arch reconstruction.
Murakami et al.: Enhanced Aortic Pressure Wave Reflection in Patients with Aortic Coarctation after Aortic Arch Repair
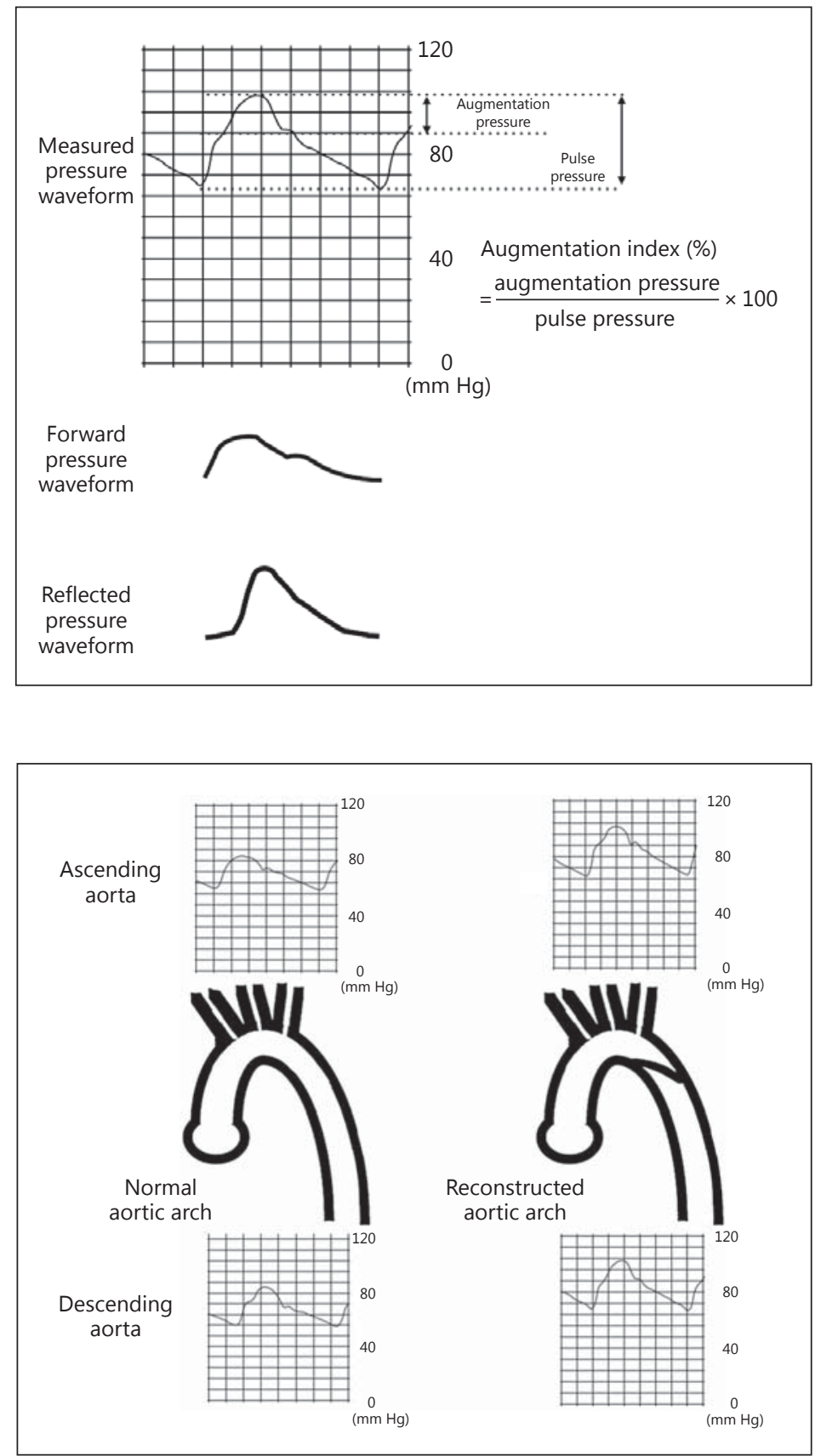

In patients after aortic coarctation repair, it is well known that the distensibility of the reconstructed portion is decreased $[15,16]$. Because wave reflections arise from discontinuities in the elastic properties along the arterial tree in which there is a change in impedance, the reconstructed aortic portion could generate a new reflection pressure wave that traveled backward toward the ascending aorta. We previously reported the characteristics of the aortic pressure waveform, which was recorded using a pressure sensor-mounted catheter, in 20 patients after aortic arch repair compared to patients with a normal aortic arch [17]. In the paper, the pressure waveform during a pullback of the catheter from the ascending to descending aorta in a patient after aortic arch reconstruction showed that the repaired site 
generates a new pressure wave reflection. Moreover, the heart rate-corrected augmentation index measured in the ascending aorta was high in comparison to that in age-matched subjects with a normal aortic arch $(28.2 \pm 17.1$ vs. $1.4 \pm 12.2 \%, p=0.0001)$, while the index measured in the descending aorta showed no difference between the patients after aortic arch repair and the control subjects (Fig. 2).

\section{Loss of Pressure Amplification in Patients after Aortic Arch Repair}

It is well known that the peripheral pulse pressure is larger than the central pulse pressure, and this phenomenon is called pulse pressure amplification [18]. It has also been reported that the low pulse pressure amplification has a negative impact on total mortality and cardiovascular events $[19,20]$. Concerning the mechanism of the pulse pressure amplification, it has been implicated that a pressure wave reflection plays an important role. As described above, the aortic pressure waveform is composed of 2 pressure waveforms and the reflected pressure wave returns earlier in a cardiac cycle at the descending aorta than at the ascending aorta. This means that the time difference between a forward and backward (the reflected pressure wave) pressure wave arrival in the descending aorta is smaller than that in the ascending aorta. As a result, the pulse pressure of the composed pressure waveform in the descending aorta is larger than that in the ascending aorta. In an aorta with arteriosclerosis, the pulse wave velocity elevates. It means an earlier return of the reflected pressure wave, and a decreased time difference between the arrival of the forward and backward pressure (the reflected pressure wave) in the ascending aorta. Therefore, the pulse pressure amplification becomes small in patients with arteriosclerosis, which is one of the reasons why the low pulse pressure amplification has a negative impact on total mortality and cardiovascular events.

Because the aorta in patients after aortic arch repair has a new pressure wave reflection point closer to the ascending aorta than in a normal aorta, the time difference between the arrival of the forward and backward pressure wave in the ascending aorta should be small in comparison with that in a normal aorta. Therefore, the ascending aortic pulse pressure in patients after aortic arch reconstruction should be wider than that in subjects with a normal aorta. This means that their pulse pressure amplification is decreased. To test this hypothesis, we measured pulse pressure in 15 children after aortic arch repair, using a pressure sensormounted catheter, and compared it with that in age-matched patients with a normal aortic arch [21]. The ascending aortic systolic blood pressure was higher (106.1 \pm 12.7 vs. $97.9 \pm$ $14.3 \mathrm{~mm} \mathrm{Hg}, p=0.015$ ) and the pulse pressure was wider ( $41.3 \pm 7.8$ vs. $36.4 \pm 5.0 \mathrm{~mm} \mathrm{Hg}$, $p=0.010$ ) in patients after aortic arch reconstruction than in the age-matched controls with a normal aortic arch, while there were no differences in those parameters in the descending aorta. In the control subjects with a normal aortic arch, the pulse pressure in the descending aorta was larger than that in the ascending aorta (pulse pressure amplification) ( $40.5 \pm 6.5$ vs. $36.4 \pm 5.0 \mathrm{~mm} \mathrm{Hg}, p<0.0001$ ). However, there was no difference in pulse pressure between the ascending and descending aorta in patients after aortic arch repair (41.3 \pm 7.8 vs. $41.0 \pm$ $7.7 \mathrm{~mm} \mathrm{Hg}$, ns). The pulse pressure amplification in the patients after aortic arch reconstruction, which was defined as the pulse pressure in the descending aorta minus that in the ascending aorta, was significantly lower than that in the control subjects with a normal aortic arch $(-0.3 \pm 1.7$ vs. $4.1 \pm 2.9 \mathrm{~mm} \mathrm{Hg}, p<0.0001)$. The loss of pulse pressure amplification in patients after aortic arch repair could be one of the causes of their frequent cardiovascular disease. 
Murakami et al.: Enhanced Aortic Pressure Wave Reflection in Patients with Aortic Coarctation after Aortic Arch Repair

\section{Enhanced Aortic Pressure Wave Reflection and Its Influence on the Left Ventricle}

As mentioned above, in patients after aortic arch repair, the reconstructed site in the aortic arch generates a new pressure wave reflection and enhances aortic pressure augmentation. We previously examined the influence of the enhancement of a pressure wave reflection on patients' left ventricles. We measured the augmentation index in 61 pediatric patients with a normal aortic arch using a pressure sensor-mounted catheter and clarified the relationship between their augmentation index and height $[14,22]$. Using this relationship, we calculated the increment of the augmentation index in 19 patients after aortic arch reconstruction [22]. The increment of the augmentation index in patients after aortic arch repair had a significant correlation with left ventricular posterior wall thickness ( $r=0.4075, p=0.0373)$. Therefore, the enhancement of the pressure wave reflection in patients after aortic arch repair could cause left ventricular hypertrophy. Moreover, we examined the serum concentration of the procollagen type III amino-terminal peptide in the patients in order to examine myocardial fibrosis [23]. In pathological left ventricular hypertrophy, myocardial fibrosis occurs. Myocardial fibrosis is quite commonly observed in patients with hypertension and one of the most important independent risk factors for cardiovascular mortality and morbidity. The procollagen type III amino-terminal peptide is one of the biomarkers for myocardial fibrosis. The serum concentration of the procollagen type III amino-terminal peptide in patients after aortic arch repair was elevated and had a significant correlation with their left ventricular mass $(r=0.63, p<0.01)$. These results suggest that left ventricular hypertrophy induced by the enhancement of the pressure wave reflection in patients after aortic arch repair leads to pathological left ventricular hypertrophy and could be one of the causes of cardiovascular diseases.

\section{Pressure Wave Refection in Patients after a Catheter Intervention}

As is evident from the above, surgical reconstruction of aortic arch could generate a new pressure wave reflection. Then, does a catheter intervention for an aortic coarctation generate a new pressure wave reflection? We previously analyzed the pressure waveforms in patients with coarctation of the aorta after balloon dilation [24]. In the paper, the augmentation index measured just proximal to the dilated portion in the aortic arch was larger than the index at the descending aorta, although there was no difference between the systolic blood pressure in the ascending and that in the descending aorta. This result means that the reconstructed portion by the balloon angioplasty could also generate a new pressure wave reflection. Further analysis of the pressure waveform in patients after balloon dilatation or stent implantation in the aortic is needed in order to clarify the pressure wave reflection of the patients after aortic coarctation after catheter interventions.

We studied the pressure waveform in patients after aortic arch repair focusing on the pressure wave reflection. Recently, researchers in other institutes have also become interested in the analysis of the pressure waveform after aortic arch reconstruction [25]. I hope many investigators will participate in the study of this area and clarify the mechanism of cardiovascular disease in patients after aortic arch repair.

\section{Disclosure Statement}

The author has no conflicts of interest to disclose. 
Murakami et al.: Enhanced Aortic Pressure Wave Reflection in Patients with Aortic

Coarctation after Aortic Arch Repair

\section{References}

-1 Presbitero P, Demarie D, Villani M, Perinetto EA, Riva G, Orzan F, Bobbio M, Morea M, Brusca A: Long term results (15-30 years) of surgical repair of aortic coarctation. Br Heart J 1987;57:462-467.

2 Stewart AB, Ahmed R, Travill CM, Newman CG: Coarctation of the aorta life and health 20-44 years after surgical repair. Br Heart J 1993;69:65-70.

3 Jenkins NP, Ward C: Coarctation of the aorta: natural history and outcome after surgical treatment. QJM 1999; 92:365-371.

4 Cohen M, Fuster V, Steele PM, Driscoll D, McGoon DC: Coarctation of the aorta. Long-term follow-up and prediction of outcome after surgical correction 1989;80:840-845.

5 Daniels SR: Repair of coarctation of the aorta and hypertension: does age matter 2001;358:89.

-6 Celermajer DS, Greaves K: Survivors of coarctation repair: fixed but not cured. Heart 2002;88:113-114.

7 Toro-Salazar OH, Steinberger J, Thomas W, Rocchini AP, Carpenter B, Moller JH: Long-term follow-up of patients after coarctation of the aorta repair. Am J Cardiol 2002;89:541-547.

-8 O'Sullivan JJ, Derrick G, Darnell R: Prevalence of hypertension in children after early repair of coarctation of the aorta: a cohort study using casual and $24 \mathrm{~h}$ blood pressure measurement. Heart 2002;88:163-166.

-9 0’Rourke MF: Arterial stiffness, systolic blood pressure, and logical treatment of arterial hypertension 1990; 15:339-347.

10 Nichols WW, Edwards DG: Arterial elastance and wave reflection augmentation of systolic blood pressure: deleterious effects and implications for therapy. J Cardiovasc Pharmacol Ther 2001;6:5-21.

11 Kingwell BA, Waddell TK, Medley TL, Cameron JD, Dart AM: Large artery stiffness predicts ischemic threshold in patients with coronary artery disease 2002;40:773-779.

$\checkmark 12$ Hayashi T, Nakayama Y, Tsumura K, Yoshimaru K, Ueda H: Reflection in the arterial system and the risk of coronary heart disease 2002;15:405-409.

13 Nichols WW, O’Rourke MF, Vlachopoulos C: McDonald's Blood Flow in Arteries, ed 6. London, Hodder Arnold, 2011.

14 Murakami T, Takeda A, Takei K, Ueno M, Yakuwa S, Yamazawa H, Furukawa T: Aortic pressure wave reflection in children. Hypertens Res 2010;33:225-228.

-15 Xu J, Shiota T, Omoto R, Zhou X, Kyo S, Ishii M, Rice MJ, Sahn DJ: Intravascular ultrasound assessment of regional aortic wall stiffness, distensibility, and compliance in patients with coarctation of the aorta. Am Heart J 1997;134:93-98.

16 Brili S, Dernellis J, Aggeli C, Pitsavos C, Hatzos C, Stefanadis C, Toutouzas P: Aortic elastic properties in patients with repaired coarctation of aorta. Am J Cardiol 1998;82:1140-3-A10.

$\checkmark 17$ Murakami T, Takeda A: Enhanced aortic pressure wave reflection in patients after repair of aortic coarctation. Ann Thorac Surg 2005;80:995-999.

18 Avolio AP, Van Bortel LM, Boutouyrie P, Cockcroft JR, McEniery CM, Protogerou AD, Roman MJ, Safar ME, Segers P, Smulyan H: Role of pulse pressure amplification in arterial hypertension: experts' opinion and review of the data. Hypertension 2009;54:375-383.

19 Benetos A, Gautier S, Labat C, Salvi P, Valbusa F, Marino F, Toulza O, Agnoletti D, Zamboni M, Dubail D, Manckoundia P, Rolland Y, Hanon O, Perret-Guillaume C, Lacolley P, Safar ME, Guillemin F: Mortality and cardiovascular events are best predicted by low central/peripheral pulse pressure amplification but not by high blood pressure levels in elderly nursing home subjects: the PARTAGE (Predictive Values of Blood Pressure and Arterial Stiffness in Institutionalized Very Aged Population) study. J Am Coll Cardiol 2012;60:1503-1511.

20 Benetos A, Thomas F, Joly L, Blacher J, Pannier B, Labat C, Salvi P, Smulyan H, Safar ME: Pulse pressure amplification a mechanical biomarker of cardiovascular risk. J Am Coll Cardiol 2010;55:1032-1037.

21 Murakami T, Shiraishi M, Nawa T, Takeda A: Loss of pulse pressure amplification between the ascending and descending aorta in patients after an aortic arch repair. J Hypertens 2017;35:533-537.

-22 Murakami T, Takeda A, Yamazawa H, Tateno S, Kawasoe Y, Niwa K: Aortic pressure wave reflection in patients after successful aortic arch repair in early infancy. Hypertens Res 2013;36:603-607.

23 Yamazawa H, Murakami T, Takeda A, Takei K, Furukawa T, Nakajima H: Serum concentration of procollagen type III amino-terminal peptide is increased in patients with successfully repaired coarctation of the aorta with left ventricular hypertrophy. Pediatr Cardiol 2015;36:555-560.

24 Murakami T, Ueno M, Takeda A, Yakuwa S: Pressure wave reflection after successful balloon dilatation of aortic coarctation. Circ J 2007;71:1821-1822.

25 Quail MA, Short R, Pandya B, Steeden JA, Khushnood A, Taylor AM, Segers P, Muthurangu V: Abnormal wave reflections and left ventricular hypertrophy late after coarctation of the aorta repair. Hypertension 2017;69: 501-509. 